\title{
Baseline Characteristics of Study Participants in the Early Life Interventions for Childhood Growth and Development in Tanzania (ELICIT) Trial
}

\author{
Tarina C. Parpia, ${ }^{1}$ Sarah E. Elwood, ${ }^{1}$ Rebecca J. Scharf, ${ }^{2}$ Joann M. McDermid, ${ }^{1}$ Anne W. Wanjuhi, ${ }^{2}$ \\ Elizabeth T. Rogawski McQuade, ${ }^{1}$ Jean Gratz, ${ }^{1}$ Erling Svensen, ${ }^{3}$ Jonathan R. Swann, ${ }^{4}$ Jeffrey R. Donowitz, ${ }^{5}$ Samwel Jatosh, ${ }^{6}$ \\ Siphael Katengu, ${ }^{6}$ Paschal Mdoe, ${ }^{6}$ Sokoine Kivuyo, ${ }^{7}$ Eric R. Houpt, ${ }^{1}$ Mark D. DeBoer, ${ }^{2}$ Estomih Mduma, ${ }^{6}$ and \\ James A. Platts-Mills ${ }^{1 *}$ \\ ${ }^{1}$ Division of Infectious Diseases and International Health, University of Virginia, Charlottesville, Virginia; ${ }^{2}$ Department of Pediatrics, University of \\ Virginia, Charlottesville, Virginia; ${ }^{3}$ Haukeland University Hospital, Bergen, Norway; ${ }^{4}$ School of Human Development and Health, Faculty of \\ Medicine, University of Southampton, Southampton, United Kingdom; ${ }^{5}$ Division of Infectious Disease, Children's Hospital of Richmond at Virginia \\ Commonwealth University, Richmond, Virginia; ${ }^{6}$ Haydom Global Health Research Centre, Haydom Lutheran Hospital, Haydom, Tanzania; \\ ${ }^{7}$ National Institute for Medical Research, Muhimbili Medical Research Centre, Dar es Salaam, Tanzania
}

\begin{abstract}
Recurrent enteric infections and micronutrient deficiencies, including deficiencies in the tryptophankynurenine-niacin pathway, have been associated with environmental enteric dysfunction, potentially contributing to poor child growth and development. We are conducting a randomized, placebo-controlled, $2 \times 2$ factorial interventional trial in a rural population in Haydom, Tanzania, to determine the effect of 1) antimicrobials (azithromycin and nitazoxanide) and/or 2) nicotinamide, a niacin vitamer, on attained length at 18 months. Mother/infant dyads were enrolled within 14 days of the infant's birth from September 2017 to September 2018, with the follow-up to be completed in February 2020. Here, we describe the baseline characteristics of the study cohort, risk factors for low enrollment weight, and neonatal adverse events (AEs). Risk factors for a low enrollment weight included being a firstborn child ( -0.54 difference in weight-for-age $z$-score [WAZ] versus other children, $95 \% \mathrm{Cl}:-0.71,-0.37)$, lower socioeconomic status $(-0.28,95 \% \mathrm{Cl}$ : $-0.43,-0.12$ difference in WAZ), and birth during the preharvest season (November to March) $(-0.22,95 \% \mathrm{Cl}$ : $-0.33,-0.11$ difference in WAZ). The most common neonatal serious AEs were respiratory tract infections and neonatal sepsis (2.2 and 1.4 events per 100 child-months, respectively). The study cohort represents a high-risk population for whom interventions to improve child growth and development are urgently needed. Further analyses are needed to understand the persistent impacts of seasonal malnutrition and the interactions between seasonality, socioeconomic status, and the study interventions.
\end{abstract}

\section{INTRODUCTION}

Poor child growth is an intractable problem in resourcepoor settings and has been associated with poor cognitive development, decreased school performance, and lower economic productivity. ${ }^{1-3}$ Haydom, Tanzania, a rural, agricultural town, was one of eight sites included in the Etiology, Risk Factors, and Interactions of Enteric Infection and Malnutrition and the Consequences for Child Health (MAL-ED) study evaluating correlates of childhood growth and development from 2009 to $2014 .{ }^{4}$ The prevalence of stunting in the Haydom MAL-ED cohort was found to be $70 \%$ at 18 months of age. ${ }^{5,6}$ Although water, sanitation, and hygiene (WASH) interventions and micro- or macronutrient supplementation are widely thought to contribute to stunting, the efficacy of interventions to improve these factors has been inconsistent or limited. ${ }^{7-11}$ There is evidence that recurrent enteric infections and micronutrient deficiencies, including deficiencies in the tryptophan-kynurenineniacin pathway, are associated with environmental enteric dysfunction, which may contribute to poor child growth and stunting. Clinically, a deficiency of niacin and the essential amino acid tryptophan may manifest as pellagra. ${ }^{2,5,12-15}$ We have previously described the rationale and design of the Early Life Interventions for Childhood Growth and Development in Tanzania (ELICIT) study, an ongoing interventional trial to determine the effect of antimicrobials and micronutrient supplementation on linear growth in children in Haydom. ${ }^{6}$

\footnotetext{
${ }^{*}$ Address correspondence to James A. Platts-Mills, Division of Infectious Diseases and International Health, University of Virginia, 345 Crispell Drive, Room 1522, Charlottesville, VA 22908-1340.
} E-mail: jp5t@virginia.edu
Because the study population in Haydom and the surrounding area live in a region with a unimodal crop cycle and a maize predominant diet, they may be particularly predisposed to nutritional deficiencies, including niacin. Seasonal patterns of food insecurity and hunger have been shown to significantly impact birth weight and rates of acute malnutrition in this area. ${ }^{16}$ Birth season, typically defined in relation to the harvest, has been associated with long-term negative consequences on growth and educational attainment in Tanzania and is more predictive of these outcomes than average food consumption. ${ }^{17}$ During the preharvest period, a time when the previous year's harvest stocks are diminished, both diet diversity and the quantity of food consumed are reduced. ${ }^{18}$ This preharvest season is also associated with seasonal peaks in Shigella infections, which have been associated with poor growth. ${ }^{15,19}$ It is, therefore, possible that the study interventions may have a greater impact in the preharvest season. Because the impact of interventions to improve child growth and development may be poorly generalizable across populations, we describe the baseline characteristics of the ELICIT study population here in detail, compare these with the MAL-ED cohort, and describe seasonal and other risk factors for low enrollment weight, used as a baseline marker of prenatal risk for poor long-term growth outcomes.

\section{MATERIALS AND METHODS}

The ELICIT design and protocol is described in detail elsewhere. ${ }^{6}$ In brief, the study is a randomized, placebo-controlled, interventional trial to determine whether administration of 1) antimicrobials (azithromycin and nitazoxanide) to reduce the subclinical carriage of enteric pathogens and/or 2) nicotinamide, a niacin vitamer, to reduce intestinal inflammation ${ }^{14}$ has 
an effect on linear growth at 18 months. Mothers could provide informed consent during pregnancy, but mother/child dyads were enrolled within 14 days of the child's birth from September 2017 to September 2018, when the inclusion and exclusion criteria were met. Specifically, participants were eligible for enrollment if the mother was 18 years of age or older and if the child was 14 days of age or younger and born in the Haydom catchment area. Participants were excluded for maternal inability to adhere to the protocol (i.e., is not able to follow directions from the field team), multiple gestation, significant birth defect or neonatal illness, weight $<1500 \mathrm{~g}$ at enrollment (age $\leq 14$ days), lack of intent to breastfeed the infant, and plan to move from the area within the subsequent 18 months. ${ }^{6}$ As a $2 \times 2$ factorial study design, dyads were randomized to one of four arms: placebo + placebo, azithromycin and nitazoxanide + placebo, placebo + nicotinamide, or azithromycin and nitazoxanide + nicotinamide. Mothers were given daily nicotinamide (or placebo) from birth through 6 months. Children received daily nicotinamide (or placebo) starting at 6 months. Azithromycin and nitazoxanide (or placebo) were given at 6, 9, 12 , and 15 months, or 12 and 15 months, respectively. In addition to receiving interventions, standardized questionnaires were administered at each monthly visit to document maternal reports of illness, healthcare-seeking behavior, infant feeding practices, and dietary information including food insecurity. A broad sociodemographic questionnaire was administered at the first monthly visit, including questions about the household composition, WASH, income, family assets, and exposure to animals. All enrolled children who completed this first visit, regardless of subsequent follow-up status, were included in this analysis.

Household characteristics were previously compiled for children enrolled in the MAL-ED study (2009-2014) from a similar geographic area. Characteristics of the households in the greater Manyara Region, in which most of the ELICIT and MAL-ED study populations reside, were compiled from the 2015-2016 Tanzania Demographic and Health Survey and Malaria Indicator Survey (DHS), a nationally representative household survey. ${ }^{20}$

Study setting. Haydom is located in the Manyara (northcentral) region of Tanzania. Haydom Lutheran Hospital, Haydom town, is the largest hospital in the Mbulu district. The study population lives primarily in the Manyara Region as well as in a small portion of the Singida region, within an approximately $25-\mathrm{km}$ radius from Haydom town (Figure 1). The area is ethnically and geographically diverse and relies primarily on subsistence agriculture. There is one annual harvest starting in approximately April of each year. ${ }^{4,15}$ Of note, the MAL-ED catchment area did not include Haydom town.

Variable definitions. Improved sanitation and drinking water sources were defined based on the WHO guidelines. ${ }^{21}$ An improved drinking water source included a household connection, public standpipe, borehole, protected dug well, protected spring, or rainwater collection. Improved sanitation facilities were connected to a public sewer or septic system, a pour-flush latrine, or a ventilated improved pit latrine. Treated water referred to water that was boiled, filtered, or treated with bleach. The Water and sanitation, Assets, Maternal education, and household Income (WAMI) score, calculated for children in both MAL-ED and ELICIT, is a composite measure of socioeconomic status derived using data from all eight MAL-ED sites. ${ }^{22}$ Crowding was defined as two or more people sleeping per room in the household.

Anthropometry was performed as previously described. ${ }^{6}$ In brief, all field team members were trained in the accurate measurement of anthropometrics and a standardized operating protocol was used for all measurement procedures. The enrollment weight, in kilograms, was measured to the nearest

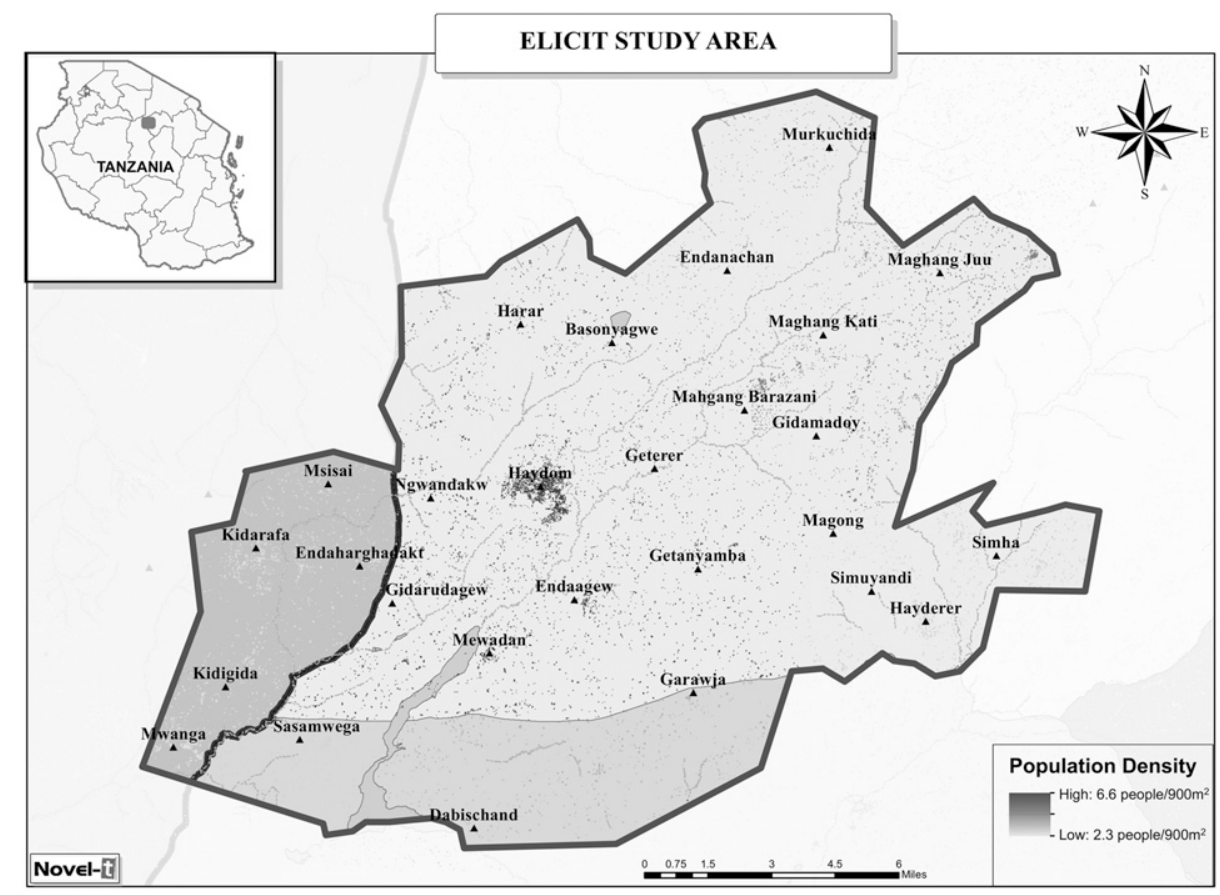

FIGURE 1. Early Life Interventions for Childhood Growth and Development in Tanzania study area. Shading and outlines indicate the three districts and two regions included in the study area (lightest shading: Mbulu/Manyara; intermediate shading: Hananga/Manyara; darkest shading: Mkalama/Singida). 
TABLE 1

Baseline characteristics in the ELICIT trial, in comparison to other studies in the same geographical region, during different time periods

\begin{tabular}{|c|c|c|c|}
\hline & ELICIT & MAL-ED & Demographic and Health Survey \\
\hline & $(2017-2018)$ & (2009-2014) & (2015-2016) \\
\hline & $(n=1,170)^{\star}$ & $(n=250) \dagger$ & $(n=426)$ \\
\hline Sociodemographics & & & \\
\hline Female gender & $573(49.0)$ & $127(50.8)$ & NA \\
\hline Affiliation with Iraqw tribe & $940(80.3)$ & $226(90.4)$ & NA \\
\hline Firstborn child & $205(17.5)$ & $24(9.6)$ & NA \\
\hline Hospital birth & $609(52.1)$ & $125(50.0)$ & NA \\
\hline Maternal age & $27.6 \pm 6.6$ & $29.9 \pm 6.7$ & NA \\
\hline Mother with $\geq 7$ years of education & $879(75.1)$ & $149(59.6)$ & $143 / 267(53.6)$ \\
\hline Monthly income (/1,000 Tanzanian shillings) & $48.8 \pm 51.3$ & $43.1 \pm 56.2$ & NA \\
\hline Risk factors & & & \\
\hline Exclusive breastfeeding in the first month & $1,105(94.4)$ & $213(85.2)$ & NA \\
\hline Access to an improved drinking water source & $778(66.5)$ & $102(40.8)$ & $355(83.3)$ \\
\hline Routine treatment of drinking water & $9(0.8)$ & $15(6.0)$ & $81(19.0)$ \\
\hline Drinking water $>10$ minutes from home & $950(81.2)$ & $243(97.2)$ & $386(90.6)$ \\
\hline Access to an improved latrine & $126(10.8)$ & $40(16)$ & $178(41.8)$ \\
\hline Crowding & $373(31.9)$ & $130(52.0)$ & NA \\
\hline Agricultural land ownership & $1,128(96.4)$ & $243(97.2)$ & $276(64.8)$ \\
\hline Chicken ownership $\ddagger$ & $984(84.1)$ & $157(62.8)$ & $230(54.0)$ \\
\hline Cow ownership§ & $793(67.8)$ & $220(88)$ & 53 (12.4) \\
\hline Household assets & & & \\
\hline Electricity & $398(34.0)$ & $2(0.8)$ & NA \\
\hline Mattress & $667(57.0)$ & $103(41.2)$ & NA \\
\hline $\begin{array}{l}\text { Table } \\
\text { Tab }\end{array}$ & $483(41.3)$ & $73(29.2)$ & NA \\
\hline Bench & $988(84.4)$ & 108 (43.2) & NA \\
\hline Separate kitchen & $725(62.0)$ & $100(40.0)$ & NA \\
\hline Refrigerator & $14(1.2)$ & $0(0)$ & $9(2.1)$ \\
\hline Television & $76(6.5)$ & $2(0.8)$ & $40(9.4)$ \\
\hline Mobile phone & $976(83.4)$ & $149(59.6)$ & $323(75.8)$ \\
\hline Family bank account & $69(5.9)$ & $11(4.4)$ & $134(31.5)$ \\
\hline Anthropometry & & & \\
\hline Enrollment weight-for-age $z$-score $\|$ & $-0.60 \pm 0.98$ & $-0.14 \pm 0.90$ & NA \\
\hline Enrollment height-for-age $z$-score & $-0.79 \pm 1.04$ & $-1.01 \pm 1.13$ & NA \\
\hline Enrollment head-circumference-for-age $z$-score & $-0.03 \pm 1.02$ & $0.16 \pm 1.07$ & NA \\
\hline $\begin{array}{l}\text { Water and sanitation, Assets, Maternal education, } \\
\text { and household Income index score (median, } \\
\text { interquartile range) } 3\end{array}$ & $0.30,0.17$ & $0.23,0.18$ & NA \\
\hline 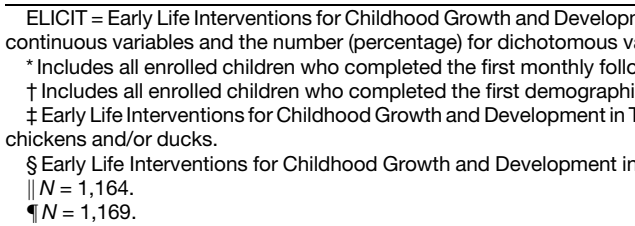 & $\begin{array}{l}\text { MAL-ED = Malnutri } \\
\text { therwise stated. } \\
\text { nnaires ask only abo } \\
\text { ionnaire asked if the }\end{array}$ & $\begin{array}{l}\text { ership, and the MAL } \\
\text { ned cows and/or go }\end{array}$ & $\begin{array}{l}\text { tavailable. Mean } \pm \text { SD is shown for } \\
\text { s ask whether the household owns }\end{array}$ \\
\hline
\end{tabular}

$10 \mathrm{~g}$ using a digital scale. Length was measured to the nearest millimeter by two research field-workers with the child lying flat on a measuring board with a fixed perpendicular board against which the head was placed and a second adjustable perpendicular board was placed against the feet. An average of two measurements was used, if within $2 \mathrm{~mm}$ of each other. If not, a third measurement was obtained, and the average of the two closest measurements was used. Head circumference, in centimeters, was measured to the nearest millimeter by placing a non-distensible tape around the child's head, above the eyebrows, above the ears, and around the biggest part of the back of the head. Weight-forage $z$-score (WAZ), length-for-age $z$-score (LAZ), and head circumference-for-age $z$-score $(\mathrm{HCZ})$ were calculated per the 2006 WHO growth standards, ${ }^{23}$ using the zscorer package version 0.2 .0 in $R .^{24} Z$ score values were then cleaned by first identifying outliers defined as values outside of the 0.5 th percentile, which were then removed if not consistent with subsequent measurements.

Exclusive breastfeeding status in the ELICIT trial, asked at the first monthly visit, was defined as current breastfeeding without any introduction of liquids or solid food into the diet in the previous month. To assess for food insecurity, mothers were asked, "Since the last visit, has the mother worried that the household would not have enough food?" Mothers could respond "No," "Rarely," "Sometimes," or "Often." We converted this into a binary indicator to compare those who had no concern over food availability with those who expressed any concern. Using data available from both the MAL-ED and ELICIT cohorts, the preharvest season was defined as the period of consistently high food insecurity, from November to March, ending with the harvest in April.

Adverse events (AEs) and serious adverse events (SAEs) that occurred in the first month of life were also recorded. An AE was defined as any untoward or unfavorable medical occurrence in a participant, including any abnormal sign (e.g., abnormal physical examination or laboratory finding), symptom (e.g., maternal report during a monthly study visit), or disease even if the event is not considered to be related to the investigational interventions. An SAE was defined as an event that resulted in any of the following outcomes: death, life-threatening illness, hospitalization or prolongation of 
hospitalization, an event that resulted in persistent or significant incapacity/disability, an event that was medically significant and which the investigator regarded as serious based on medical judgment, or a laboratory result on the safety laboratory test that was in the range for SAE. All SAE reports were reviewed by a pediatrician to ensure appropriate event categorization.

Statistical analysis. Baseline characteristics were reported using frequencies and means. Summary statistics for major socioeconomic and demographic indicators were tabulated for ELICIT, MAL-ED, and the DHS where questions were comparable enough to be reasonably certain the data were capturing the same classification. Univariable and multivariable linear regression models were performed to assess factors influencing enrollment WAZ and LAZ. However, pre- and postharvest seasons were used rather than individual calendar month to the multivariable model. Poisson approximation to log-binomial regression with robust standard errors was used to estimate risk of AEs and SAEs. Statistical analyses were performed using $R$ version 3.5.2.

\section{RESULTS}

In total, 1,205 children were assessed for eligibility, of whom $1,188(98.6 \%)$ were enrolled and 1,170 (97.1\%) completed the first monthly visit. In comparison to a previous cohort study conducted in the same region (MAL-ED), there was evidence of socioeconomic improvements in the population (Table 1). In ELICIT, a higher proportion of mothers had greater than or equal to 7 years of education (15.5\% increase, $95 \% \mathrm{Cl}: 9.1 \%$,
$22.1 \%$ ) and reported exclusive breastfeeding in the first month (9.2\% increase, $95 \% \mathrm{Cl}: 5.1 \%, 14.3 \%)$. In addition, more households reported an improved drinking water source in ELICIT (25.7\% increase, 95\% Cl: 19.0\%, 32.2\%), although a decreased proportion reported access to an improved latrine (5.2\% decrease, $95 \% \mathrm{Cl}: 0.7 \%, 10.5 \%)$. A higher proportion of households in ELICIT reported owning assets such as benches, mattresses, and mobile phones; having electricity; and having a separate kitchen. The mean enrollment WAZ and $\mathrm{HCZ}$ in ELICIT were significantly lower than those on the MALED population (-0.46 difference, $95 \% \mathrm{Cl}$ : $-0.59,-0.33$ and -0.19 difference, $95 \% \mathrm{Cl}:-0.33,-0.05$, respectively). On the other hand, the mean LAZ enrollment for ELICIT was significantly higher than that in MAL-ED (0.21 difference, $95 \% \mathrm{Cl}$ : $0.07,0.35)$. In comparison to the DHS from the Manyara Region, there was a decrease in improved access to drinking water, sanitation, and assets compared with the households in the Haydom catchment area.

Total rainfall in the year before the ELICIT enrollment period (2017-2018) was particularly low and short (Figure 2), leading to a poorer harvest. We estimated the impact of birth season and other risk factors for enrollment WAZ as a baseline marker or prenatal risk for poor long-term growth outcomes. There was striking seasonality in enrollment WAZ (Table 2), with a nadir in December (-0.64 difference in WAZ from July; 95\% $\mathrm{Cl}$ : $-0.89,-0.38)$. In univariable analysis, birth during the preharvest season was associated with a lower enrollment WAZ (difference in enrollment WAZ for preharvest vs. postharvest: $-0.22 ; 95 \% \mathrm{Cl}:-0.33,-0.11$ ) although birth during the pre-harvest season had no association with enrollment LAZ
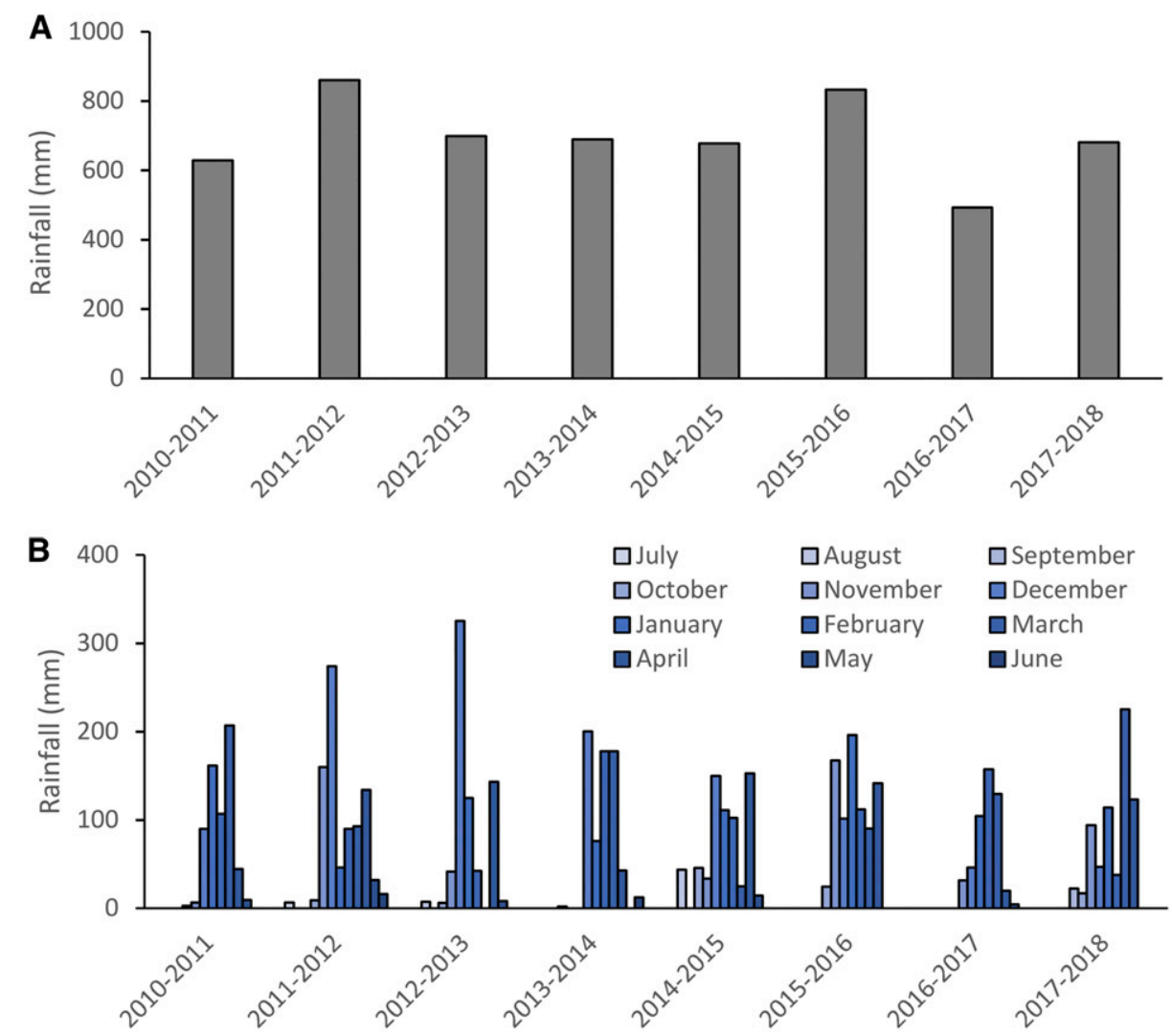

FIGURE 2. Total seasonal rainfall (A) and rainfall by calendar month and year (B) in Haydom, Tanzania. This figure appears in color at www.ajtmh.org. 
TABLE 2

Sociodemographic factors associated with enrollment WAZ

\begin{tabular}{|c|c|c|c|c|}
\hline & \multicolumn{2}{|c|}{ Difference in enrollment WAZ (95\% Cl) } & \multicolumn{2}{|c|}{ Difference in length-for-age $z$-score $(95 \% \mathrm{Cl})$} \\
\hline & Univariable & Multivariable & Univariable & Multivariable \\
\hline \multicolumn{5}{|l|}{ Calendar month } \\
\hline January & $-0.33(-0.58,-0.08)$ & $-0.37(-0.61,-0.12)$ & $0.11(-0.15,0.38)$ & $0.11(-0.15,0.37)$ \\
\hline February & $-0.32(-0.58,-0.06)$ & $-0.36(-0.61,-0.10)$ & $0.26(-0.02,0.53)$ & $0.26(-0.01,0.53)$ \\
\hline March & $-0.34(-0.60,-0.09)$ & $-0.37(-0.62,-0.11)$ & $0.01(-0.27,0.28)$ & $0.00(-0.27,0.27)$ \\
\hline April & $-0.31(-0.58,-0.03)$ & $-0.31(-0.58,-0.04)$ & $-0.13(-0.42,0.16)$ & $-0.10(-0.39,0.19)$ \\
\hline May & $-0.16(-0.44,0.13)$ & $-0.13(-0.4,0.15)$ & $-0.02(-0.31,0.28)$ & $0.04(-0.26,0.33)$ \\
\hline June & $-0.20(-0.50,0.11)$ & $-0.22(-0.52,0.08)$ & $-0.01(-0.33,0.31)$ & $0.00(-0.32,0.31)$ \\
\hline July & Ref & Ref & Ref & Ref \\
\hline August & $-0.05(-0.37,0.27)$ & $-0.05(-0.36,0.27)$ & $-0.08(-0.41,0.26)$ & $-0.04(-0.37,0.30)$ \\
\hline September & $-0.35(-0.76,0.07)$ & $-0.34(-0.74,0.06)$ & $0.69(0.25,1.13)$ & $0.67(0.24,1.10)$ \\
\hline October & $-0.47(-0.78,-0.16)$ & $-0.50(-0.80,-0.20)$ & $0.48(0.15,0.81)$ & $0.49(0.17,0.82)$ \\
\hline November & $-0.43(-0.73,-0.13)$ & $-0.45(-0.75,-0.16)$ & $0.08(-0.24,0.39)$ & $0.08(-0.23,0.39)$ \\
\hline December & $-0.64(-0.90,-0.38)$ & $-0.62(-0.87,-0.37)$ & $0.13(-0.15,0.40)$ & $0.15(-0.12,0.42)$ \\
\hline Maternal age (years) ${ }^{\star}$ & $0.11(0.07,0.15)$ & $0.03(-0.01,0.08)$ & $0.13(0.08,0.17)$ & $0.09(0.04,0.14)$ \\
\hline Female gender & $-0.06(-0.17,0.06)$ & - & $0.06(-0.06,0.17)$ & - \\
\hline Firstborn & $-0.55(-0.69,-0.40)$ & $-0.54(-0.71,-0.37)$ & $-0.39(-0.55,-0.23)$ & $-0.24(-0.42,-0.06)$ \\
\hline Born in hospital & $0.10(-0.02,0.21)$ & - & $0.10(-0.02,0.22)$ & - \\
\hline Monthly $<50,000$ Tanzanian shillings & $-0.09(-0.22,0.05)$ & - & $0.06(-0.08,0.21)$ & - \\
\hline Maternal education $\geq 7$ years & $0.09(-0.04,0.22)$ & - & $0.02(-0.12,0.16)$ & - \\
\hline Improved sanitation & $0.04(-0.01,0.08)$ & - & $-0.03(-0.08,0.02)$ & - \\
\hline \multicolumn{5}{|l|}{$\begin{array}{l}\text { Water/sanitation, Assets, Maternal } \\
\text { education, and Income score }\end{array}$} \\
\hline Fourth quartile & Ref & Ref & Ref & Ref \\
\hline Third quartile & $-0.11(-0.26,0.04)$ & $-0.09(-0.24,0.06)$ & $0.00(-0.16,0.16)$ & $0.00(-0.16,0.16)$ \\
\hline Second quartile & $-0.08(-0.25,0.09)$ & $-0.08(-0.25,0.08)$ & $-0.15(-0.33,0.03)$ & $-0.15(-0.33,0.03)$ \\
\hline First quartile & $-0.24(-0.39,-0.08)$ & $-0.28(-0.43,-0.12)$ & $-0.13(-0.30,0.03)$ & $-0.17(-0.33,0.00)$ \\
\hline Food insecurity & $-0.03(-0.17,0.12)$ & - & $0.13(-0.02,0.28)$ & - \\
\hline
\end{tabular}

${ }^{*}$ Per 5 -year increase in maternal age.

(0.04; 95\% Cl: $-0.08,0.16)$. Increasing maternal age (difference per year: $0.02,95 \% \mathrm{Cl}: 0.01,0.03$ ) and highest quartile WAMI socioeconomic status score compared with lowest quartile $(0.24,95 \% \mathrm{Cl}: 0.09,0.40)$ were associated with higher WAZ scores. On the other hand, being firstborn was associated with a lower WAZ $(-0.55,95 \% \mathrm{Cl}$ : $-0.59,-0.40)$. There was no association between household food insecurity and enrollment WAZ (-0.02, 95\% Cl: $-0.17,0.12)$. Adjusting for birth month, maternal age, WAMI score, and birth order, increasing maternal age was no longer a significant protective factor for enrollment WAZ, although the association with the other factors was not significantly changed (Table 2). Being firstborn and lower maternal age were associated with lower enrollment $L A Z$. The relationship between birth season and enrollment WAZ varied by WAMI (Figure 3), such that the lowest WAMI score quartile had low WAZ irrespective of birth season, whereas the upper quartiles showed more variation by birth season.

Adverse events and SAEs that occurred in the first month of life are shown in Table 3. A child may have had multiple events and each were documented. As expected, the most common AEs were acute lower respiratory infection (ALRI; 38\%) followed by upper respiratory tract infection (15\%). The most common SAEs were neonatal sepsis (47\%) and ALRI (32\%).

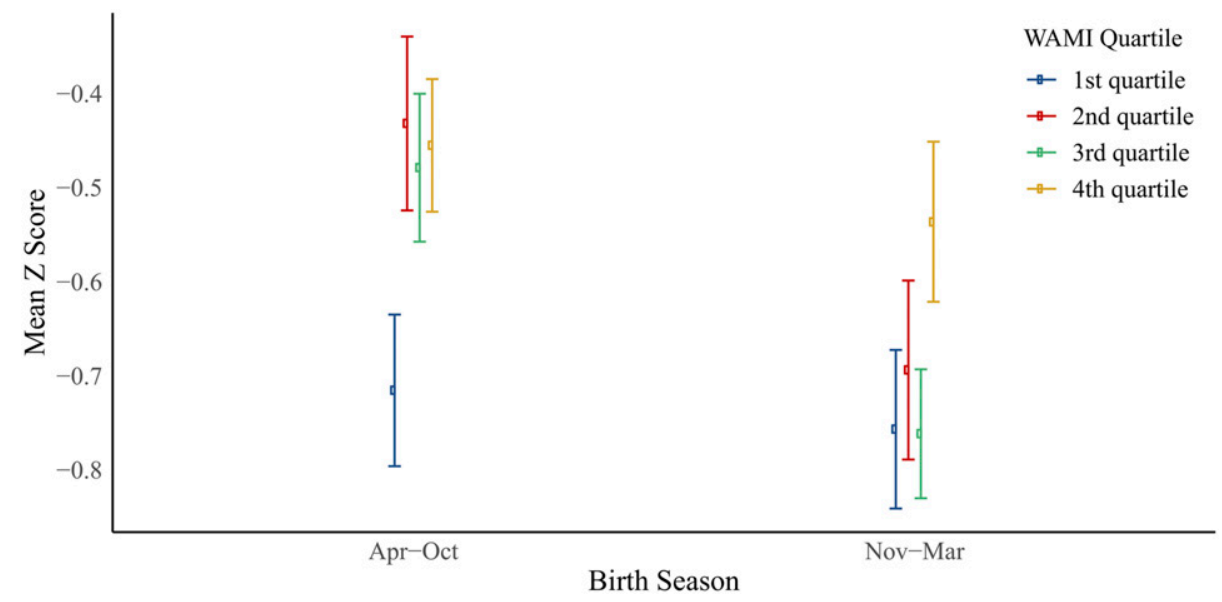

FIGURE 3. Association between birth season, socioeconomic status as measured by the Water/sanitation, Assets, Maternal education, and Income (WAMI) score, and enrollment weight-for-age $z$-score. This figure appears in color at www.ajtmh.org. 
TABLE 3

Adverse events in the first month of life in Early Life Interventions for Childhood Growth and Development in Tanzania subjects

\begin{tabular}{lcr}
\hline & AEs & Serious AEs \\
\cline { 2 - 3 } \multicolumn{1}{c}{ Event type } & $N$ (events per 100 child-months) & $N$ (events per 100 child-months) \\
\hline Any & $198(16.9)$ & $26(2.2)$ \\
Acute lower respiratory tract infection & $83(7.1)$ & $11(0.9)$ \\
Death & $5(0.4)$ & $5(0.4)$ \\
Diarrhea & $21(1.8)$ & $2(0.2)$ \\
Deep vein thrombosis & $1(0.1)$ & $0(0)$ \\
Dysentery & $1(0.1)$ & $0(0)$ \\
Fever & $4(0.3)$ & $0(0)$ \\
Fungal infection & $1(0.1)$ & $0(0)$ \\
Headache & $4(0.3)$ & $0(0)$ \\
Jaundice & $1(0.1)$ & $1(0.1)$ \\
Laboratory abnormality & $1(0.1)$ & $0(0)$ \\
Omphalitis & $5(0.4)$ & $1(0.1)$ \\
Ophthalmological illness & $8(0.7)$ & $0(0)$ \\
Other & $22(1.9)$ & $0(0)$ \\
Partial intestinal obstruction & $2(0.2)$ & $0(0)$ \\
Sepsis & $20(1.7)$ & $16(1.4)$ \\
Skin rash/infection & $12(1)$ & $2(0.2)$ \\
Upper respiratory tract infection & $33(2.8)$ & $1(0.1)$ \\
Urinary tract infection & $0(0)$ & $0(0)$ \\
\hline
\end{tabular}

$\mathrm{AEs}=$ adverse events.

Five neonatal deaths occurred among mothers who had signed consent during pregnancy but before infant enrollment. In univariate analysis, there were no strong risk factors for AEs or SAEs (Table 4). Birth during the preharvest months, lower maternal education, and lower WAMI score all were associated with increased risk of AEs and SAEs, but estimates were imprecise, given low number of events.

\section{DISCUSSION}

We identified several risk factors for enrollment weight in this population used as a marker of prenatal malnutrition. First, in a low-income setting with a unimodal crop cycle, there was a prominent seasonality to birth weight, with lower enrollment weights during the preharvest months. This was more pronounced in households above the first WAMI quartile. These results were also demonstrated in the MAL-ED cohort study where children born during the preharvest months of December to February had a statistically significant decrease in enrollment weight compared with other months. ${ }^{15}$ Maternal weight gain in the second and third trimesters has been previously shown to significantly increase birth weight in a similar setting. ${ }^{25}$ The strong association in our study between the preharvest season and low enrollment weight suggests that maternal nutrition during the third trimester of gestation is particularly critical. Increased diet diversity and nutritional counseling have also been shown to have a positive impact on

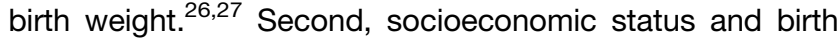
order were associated with enrollment weight. These factors could modify the impact of the study interventions on child growth and developmental outcomes.

There are potential long-term implications of low birth weight. The prevalence of malnutrition (stunting, wasting, and underweight) is strikingly higher in children with low birth weight, ${ }^{28}$ and these anthropometric measurements correlate with cognitive performance in these and other settings. ${ }^{29}$ This study, in which child growth and development were assessed in a large birth cohort, will provide an opportunity to define the long-term impact of birth season and enrollment weight or both growth and cognitive development. Although the primary outcome of the ELICIT trial is attained length at 18 months, the effect of postnatal malnutrition on length lags behind effects on

TABLE 4

Risk factors for AEs in the first month of life in Early Life Interventions for Childhood Growth and Development in Tanzania

\begin{tabular}{lll}
\hline \multicolumn{1}{c}{ Risk factor } & \multicolumn{1}{c}{ AEs } & Serious AEs \\
\cline { 2 - 3 } & Risk ratio [95\% Cl] \\
\hline During preharvest season (November-March) & $1.22(0.91,1.66)$ & $1.96(0.80,5.49)$ \\
Weight-for-age $z$-score at enrollment & $0.99(0.85,1.15)$ & $0.76(0.52,1.17)$ \\
Firstborn child & $0.97(0.64,1.40)$ & $2.35(0.89,5.66)$ \\
Income $<50,000$ Tanzanian shillings & $0.97(0.69,1.40)$ & $0.91(0.36,2.77)$ \\
Maternal education $\geq 7$ years & $0.74(0.55,1.03)$ & $0.44(0.19,1.08)$ \\
Improved sanitation & $1.03(0.92,1.15)$ & $1.18(0.86,1.51)$ \\
Water/sanitation, Assets, Maternal education, and Income score & $0.86(0.59,1.27)$ & $1.09(0.36,3.37)$ \\
$\quad$ Third quartile & $0.70(0.43,1.12)$ & $1.01(0.26,3.54)$ \\
Second quartile & $1.10(0.76,1.62)$ & $0.75(0.19,2.63)$ \\
$\quad$ First quartile & $0.89(0.65,1.24)$ & $0.60(0.26,1.53)$ \\
Chicken ownership & $1.14(0.80,1.60)$ & $0.92(0.26,2.49)$ \\
Pig ownership & $0.89(0.67,1.2)$ & $0.72(0.28,1.70)$ \\
Cow ownership & & \\
\hline AEs = adverse events. & &
\end{tabular}


weight $^{30}$ and thus may be a less sensitive indicator of in utero insult or growth potential at time of birth. Notably, September and October had the highest enrollment lengths, consistent with a 2- to 3-month lag from the highest enrollment weights.

Despite the evidence of sociodemographic improvement in ELICIT compared with the MAL-ED, enrollment WAZ and HCZ were significantly lower. This could be due to the particularly poor harvest that preceded ELICIT enrollment. If seasonal malnutrition was particularly prominent during ELICIT enrollment, this may impact the generalizability of the interventions to other years, even in the same study area. Water, sanitation, and hygiene status also worsened from the MAL-ED to ELICIT, and access to improved WASH was substantially lower in the Haydom area than that in the Manyara region. This, in addition to high exposure to zoonotic pathogens associated with animal husbandry, may increase the force of enteric infections compared with other areas; therefore, the impact of scheduled, interval antibiotic exposure may be weaker than that in areas with a lower force of infection. As noted, the MAL-ED catchment area did not include the more populated Haydom town which may partially account for the secular changes noted between studies.

Interestingly, whereas at the population level, food insecurity was strikingly seasonal (data not shown), at the individual level, there was no association between food insecurity and low enrollment weight. This population-level association was also observed in the MAL-ED cohort. ${ }^{16}$ However, in the MAL-ED, food insecurity was only assessed every 6 months; therefore, individual associations could not be assessed. This suggests that although food insecurity may be a relevant metric of food availability at the population level and, thus, an indicator of seasonal variation in food availability, it may not correlate with food availability at the individual level. For example, worry in this cohort may be a measure of the increased awareness of difficulty accessing food and the resultant motivation to improve it. More specific data on food availability and intake may help further stratify risk factors for low-weight infants.

The AEs and SAEs in the first month occurred before any antibiotic intervention and when mothers had recently begun taking nicotinamide or placebo. As such, these events are likely reflective of the baseline risk in this population. Further analysis of the AEs and SAEs recorded throughout the study period will allow us to assess for any association with the primary interventions and will provide more power to understand the impact of birth season on childhood illness.

Our study has limitations. First, the difference between MAL-ED, ELICIT, and the regional DHS may reflect both socioeconomic improvements and the difference in the study areas, making it difficult to identify the precise reason for any observed differences. Second, defining the preharvest season may vary as this was contingent on food yields from the prior harvest and the timing of the subsequent harvest. We have used a restrictive definition of the preharvest season based on several years of data from the study area, but there may be some misclassification of birth season from year to year. As the derivation of this window is not standardized, it can be difficult to compare across studies. Third, the WAMI score was created from eight diverse sites and may not be the optimal metric for this specific area. ${ }^{22}$ Finally, the categories for AEs were defined by physician review, but broadly, the diagnostic capacity is limited. Furthermore, the power to detect risk factors for early AEs was limited by low event rates.
In summary, the ELICIT cohort was designed to probe potential pathways between early enteric infections, micronutrient availability, and child growth outcomes (LAZ). The study population has substantial variation in baseline characteristics and nutritional status driven by birth season, lower socioeconomic status, and birth order. Further analysis is needed to understand the long-term impacts of seasonal malnutrition, the potential value of maternal interventions to improve low birth weight, and the interactions between these risk factors and the study interventions.

Received December 10, 2019. Accepted for publication June 21, 2020.

Published online August 10, 2020.

Acknowledgment: We thank the research staff and participants for their important contributions.

Financial support: This work is supported by the Bill \& Melinda Gates Foundation, OPP1141342 to E. M.; NIH 5T32AI007046-43 to T. C. P.; DDCF CSDA 2014113 to R. J. S.

Authors' addresses: Tarina C. Parpia, Sarah E. Elwood, Rebecca J. Scharf, Joann M. McDermid, Anne W. Wanjuhi, Elizabeth T. Rogawski McQuade, Jean Gratz, Eric R. Houpt, Mark D. DeBoer, and James A. Platts-Mills, University of Virginia, Charlottesville, VA, E-mails: tcp7df@virginia.edu, see4v@hscmail.mcc.virginia.edu, rs3yk@ hscmail.mcc.virginia.edu, joann.mcdermid@virginia.edu, aww3y@ hscmail.mcc.virginia.edu, etr5m@virginia.edu, jean.gratz@gmail.com, erh6k@virginia.edu, mdd5z@hscmail.mcc.virginia.edu, and jp5t@ virginia.edu. Erling Svensen, Haukeland University Hospital, Bergen, Norway, E-mail: erling.svensen@helse-bergen.no. Jonathan R. Swann, University of Southampton, Southampton, United Kingdon, E-mail: j.swann@imperial.ac.uk. Jeffrey R. Donowitz, Virginia Commonwealth University, Richmond, VA, E-mail: jeffrey.donowitz@ vcuhealth.org. Samwel Jatosh, Siphael Katengu, Paschal Mdoe, and Estomih Mduma, Haydom Global Health Institute, Haydom, Tanzania, E-mails: toshsamy@gmail.com, cfranka5@gmail.com, pfmdoe@gmail. com, and esto@haydom.co.tz. Sokoine Kivuyo, National Institute for Medical Research, Muhimbili Centre, Dar es Salaam, Tanzania, E-mail: sokoinele@gmail.com.

This is an open-access article distributed under the terms of the Creative Commons Attribution (CC-BY) License, which permits unrestricted use, distribution, and reproduction in any medium, provided the original author and source are credited.

\section{REFERENCES}

1. UNICEF, World Health Organization, World Bank, 2018. Joint Child Malnutrition Estimates. Available at: http://datatopics. worldbank.org/child-malnutrition. Accessed December 10, 2019.

2. Black RE et al., 2013. Maternal and child undernutrition and overweight in low-income and middle-income countries. Lancet 382: 427-451.

3. Heckman JJ,2006. Skill formation and the economics of investing in disadvantaged children. Science 312: 1900-1902.

4. Mduma $E$ et al., 2014. The etiology, risk factors, and interactions of enteric infections and malnutrition and the consequences for child health and development study (MAL-ED): description of the Tanzanian site. Clin Infec Dis 59: S325S330.

5. MAL-ED Network Investigators, 2017. Childhood stunting in relation to the pre- and postnatal environment during the first 2 years of life: the MAL-ED longitudinal birth cohort study. PLOS Med 14: e1002408.

6. DeBoer MD et al., 2018. Early life interventions for childhood growth and development in Tanzania (ELICIT): a protocol for a randomized factorial, double-blind, placebo-controlled trial of azithromycin, nitazoxanide, and nicotinamide. BMJ Open 8: e021817. 
7. Luby SP et al., 2018. Effects of water quality, sanitation, handwashing, and nutritional interventions on diarrhea and child growth in rural Bangladesh: a cluster randomised control trial. Lancet Glob Health 6: e302-e315.

8. lannotti LL et al., 2017. Eggs in early complementary feeding and child growth: a randomized controlled trial. Pediatrics 140: e20163459.

9. Stewart CP, Caswell B, lannotti L, Lutter C, Arnold CD, Chipatala $\mathrm{R}$, Prado EL, Maleta K, 2019. The effect of eggs on early child growth in rural Malawi: the Mazira Project randomized controlled trial. Am J Clin Nutr 10: 1026-1033.

10. Dewey KG, Mridha MK, Arnold CD, Cummins JR, Khan MS, MaaloufManasseh Z, Siddiqui Z, Ullah MB, Vosti SA, 2017. Lipid-based nutrient supplementation in the first $1000 \mathrm{~d}$ improves child growth in Bangladesh: a cluster-randomized effectiveness trial. Am J Clin Nutr 105: 944-957.

11. Prendergast AJ, Humphrey JH, 2014. The stunting syndrome in developing countries. Paediatr Int Child Health 34: 250-265.

12. SembaRD, Shardell M, Trehan I, Moaddel R, Maleta KM, Ordiz MI, Kraemer K, Khadeer M, Ferrucci L, Manary MJ, 2016. Metabolic alterations in children with environmental enteric dysfunction. Sci Rep 6: 28009.

13. Le Floc'h N, Otten W, Merlot E, 2011. Tryptophan metabolism, from nutrition to potential therapeutic options. Amino Acids 41: 1195-1205.

14. Perlot T, Penninger JM, 2013. ACE 2 - from the renin-angiotensin system to gut microbiota and malnutrition. Microbes Infect 15: 866-873.

15. Rogawski ET et al., 2018. Use of quantitative molecular diagnostic methods to investigate the effect of enteropathogens infections on linear growth in children in low-resource settings: longitudinal analysis of results from the MAL-ED cohort study. Lancet Glob Health 6: e1319-e1328.

16. Rogawski McQuade ET et al., 2019. Seasonal food insecurity in Haydom, Tanzania is associated with low birthweight and acute malnutrition: results from the MAL-ED study. Am J Trop Med Hyg 100: 681-687.

17. Christian P, Dillon B, 2018. Growing and learning when consumption is seasonal; long-term evidence from Tanzania. Demography 55: 1091-1118.

18. Vaitla B, Devereux S, Swan SH, 2009 Seasonal hunger: a neglected problem with proven solutions. PLoS Med 6: e1000101.

19. Platts-Mills JA et al., 2015. Pathogen-specific burdens of community diarrhoea in developing countries: a multisite birth cohort study (MAL-ED). Lancet Glob Health 3: e564-e575.

20. Ministry of Health, Community Development, Gender, Elderly and Children (MoHCDGEC) [Tanzania Mainland], Ministry of Health
(MoH) [Zanzibar], National Bureau of Statistics (NBS), Office of the Chief Government Statistician (OCGS), and ICF, 2016. Tanzania Demographic and Health Survey and Malaria Indicator Survey (TDHS-MIS) 2015-16. Dar es Salaam, Tanzania, and Rockville, MA: MoHCDGEC, MoH, NBS, OCGS, and ICF. Available at: https:// dhsprogram.com/publications/Recommended-Citations.cfm. Accessed December 10, 2019.

21. World Health Organization and United Nations Children's Fund, 2004. Meeting the MDG Drinking Water and Sanitation Target: A Mid-Term Assessment of Progress. Geneva, Switzerland: WHO. Available at: https://www.who.int/water_sanitation_health/ monitoring/jmp04.pdf. Accessed December 10, 2019.

22. Psaki SR et al., 2014. Measuring socioeconomic status in multicountry studies: results from the eight-country MAL-ED study. Popul Health Metr 12: 8.

23. World Health Organization, 2006. WHO Child Growth Standards: Length/Height-for-Age, Weight-for-Age, Weight-for-Length, Weight-for-Height and Body Mass Index-for-Age. Geneva, Switzerland: WHO. Available at: https://www.who.int/childgrowth/ standards/Technical_report.pdf.

24. Myatt M, Guervarra E, 2019. Package 'zscorer'. Available at: https://cran.r-project.org/web/packages/zscorer/zscorer.pdf.

25. Nyaruhucha CNM, Msuya JM, Ngowi B, Gimbi DM, 2006. Maternal weight gain in second and third trimesters and their relationship with birth weights in Morogoro municipality, Tanzania. Tanzania Health Res Bull 8: 41-44.

26. Zerfu T, Umeta M, Baye K, 2016. Dietary diversity during pregnancy is associated with reduced risk of maternal anemia, preterm delivery, and low birth weight in a prospective cohort study in rural Ethiopia. Am J Clin Nutr 103: 1482-1488.

27. Akter SM, Roy SK, Thakur SK, Sultana M, Khatun W, Rahman R, Saliheen SS, Alam N, 2012. Effects of third trimester counseling on pregnancy weight gain, birthweight, and breastfeeding among urban poor women in Bangladesh. Food Nutr Bull 33: 194-201.

28. Rahman MS, Howlader T, Masud MS, Rahman ML, 2016. Association of low-birth weight with malnutrition in children under five years in Bangladesh: do mother's education, socioeconomic status, and birth interval matter? PLoS One 11: e0157814.

29. Scharf RJ et al., 2018. Early childhood growth and cognitive outcomes: findings from the MAL-ED study. Matern Child Nutr 14: e12584.

30. Brown KH, Black RE, Becker S, 1982. Seasonal changes in nutritional status and the prevalence of malnutrition in a longitudinal study of young children in rural Bangladesh. Am J Clin Nutr 26: 303-313. 